\title{
EXISTENCE RESULTS FOR NONSMOOTH SECOND-ORDER DIFFERENTIAL INCLUSIONS, CONVERGENCE RESULT FOR A NUMERICAL SCHEME AND APPLICATION TO THE MODELING OF INELASTIC COLLISIONS
}

\author{
FRÉDÉRIC BERNICOT \\ CNRS - Université Lille 1, \\ Laboratoire Paul Painlevé, \\ 59655 Villeneuve d'Ascq Cedex, France \\ frederic.bernicot@math.univ-lille1.fr \\ ALINE LEFEBVRE-LEPOT \\ CNRS - Ecole Polytechnique \\ CMAP, 91128 Palaiseau Cedex, France \\ aline.lefebvre@polytechnique.edu
}

Received 9 March 2010

Revised 4 November 2010

\begin{abstract}
We are interested in the existence results for second-order differential inclusions, involving finite number of unilateral constraints in an abstract framework. These constraints are described by a set-valued operator, more precisely a proximal normal cone to a timedependent set. In order to prove these existence results, we study an extension of the numerical scheme introduced in [10] and prove a convergence result for this scheme.
\end{abstract}

Keywords: Second-order differential inclusions; proximal normal cone; inelastic collisions; numerical scheme.

AMS Subject Classification: 34A60, 34A12, 65L20

\section{Introduction}

We consider second-order differential inclusions, involving proximal normal cones. These were firstly treated by Schatzman [26] in the framework of elastic impacts and later by Moreau [15, 16] to model inelastic impacts for a mechanical system in order to describe contact dynamics. The impact law describing the dynamics leads to a nonincreasing kinetic energy at impacts. These second-order problems appear in several models of mechanical systems with a finite number of degrees of freedom and dealing with frictionless and inelastic contacts.

Let us specify this class of problems. Let $I$ be a bounded time-interval, $f: I \times \mathbb{R}^{d} \rightarrow \mathbb{R}^{d}$ be a map and $Q: I \rightrightarrows \mathbb{R}^{d}$ be a multi-valued map. The main 
question concerns the existence for solutions to the following second-order differential inclusion:

$$
\left\{\begin{array}{l}
\forall t \in I, \quad q(t) \in Q(t) \\
\frac{d^{2} q}{d t^{2}}+N(Q(\cdot), q(\cdot)) \ni f(\cdot, q(\cdot)), \\
\forall t \in I, \quad \dot{q}\left(t^{+}\right)=P_{\mathcal{C}_{t, q(t)}} \dot{q}\left(t^{-}\right) \\
q(0)=q_{0} \in \operatorname{int}[Q(0)], \\
\dot{q}(0)=u_{0} .
\end{array}\right.
$$

We denote by $\operatorname{int}[Q(0)]$ the interior of the set $Q(0)$, by $N$ the proximal normal cone and for $q \in Q(t)$, by $\mathcal{C}_{t, q}$ the set of feasible velocities:

$$
\mathcal{C}_{t, q}:=\{u, q+\epsilon u \in Q(t+\epsilon) \text { for small enough } \epsilon>0\} .
$$

We refer the reader to [4] and [5] for details concerning different normal cones ("limiting cone", "Clarke cone", ... ). Here we will deal with "uniform prox-regular sets" $Q(t)$ so, according to [25], all these cones coincide.

Remark 1.1. We are looking for solutions $q$ such that $\dot{q}$ has a bounded variation, in order that the second-order differential equation in (1.1) should be thought in the distributional sense. More precisely, we will solve it for time-measure $\dot{q} \in B V(I)$ and it should be written with time-measures

$$
d \dot{q}+N(Q(\cdot), q(\cdot)) d t \ni f(\cdot, q(\cdot)) d t .
$$

In all this work, the second-order differential inclusion will be written in the distributional sense for easiness. However, we emphasize that we consider time-measures.

This differential inclusion can be thought as follows: the point $q(t)$, submitted to the external force $f(t, q(t))$, has to live in the set $Q(t)$ and so to follow its timeevolution. The unilateral constraint " $q(t) \in Q(t)$ " may lead to some discontinuities for the velocity $\dot{q}$. For example, frictionless impacts can be modeled by a secondorder differential inclusion involving the proximal normal cone (see $[15,16])$. This differential inclusion does not uniquely define the evolution of the velocity during an impact. To complete the description, we impose the impact law

$$
\dot{q}\left(t^{+}\right)=P_{\mathcal{C}_{t, q(t)}} \dot{q}\left(t^{-}\right),
$$

introduced by Moreau in [15] and justified by Paoli and Schatzman in [19, 21] (using a penalty method) for inelastic impacts.

The set $Q(t)$ corresponds to a set of "admissible configurations" for $q$. In physical problems, it is generally described by several constaints $\left(g_{i}\right)_{i}$ as follows:

$$
Q(t):=\bigcap_{i=1}^{p}\left\{q, g_{i}(t, q) \geq 0\right\} .
$$

The existence of a solution for such second-order problems is still open in a general framework. The first positive results were obtained by Monteiro Marques [12] 
and Paoli and Schatzman [20] in the case of a smooth time-independent admissible set (which locally corresponds to the single constraint case $p=1$ in (1.3)). The proof relies on a numerical method involving a time-discretization of (1.1) in order to compute approximate solutions and is based on the study of its convergence. The multi-constraint case with analytical data was then treated by Ballard with a different method in [1], where a positive result of uniqueness for such problems was obtained. Then in [22], an existence result is proved in the case of a nonsmooth convex time-independent admissible set (given by multiple constraints). There, the active constraints are assumed to be linearly independent in the following sense: for each configuration $q \in \partial Q$, the gradients $\left(\nabla g_{i}(q)\right)_{i \in I}$ associated to active constraints $I:=\left\{i, g_{i}(q)=0\right\}$ are assumed to be linearly independent.

In the case of nonconvex admissible sets, some results were obtained for a single constraint $p=1$ (for example in [7, 8] or in [13] and [28] for the first result concerning time-dependent constraints) and very recently in $[23,24]$ for the multiconstraint case under the linear independence of the active gradients. Moreover in [10], Maury has proposed a numerical scheme for time-independent multiple and convex constraints $g_{i}$. The admissible set $Q$ is not assumed to be convex, however at each time step, the numerical scheme uses a local convex approximation of $Q$. This improvement is interesting as it permits to define an implementable scheme, since the projection onto a convex set can be performed with efficient algorithms.

A first result of convergence for this scheme was proved in [10] for a single constraint and applications to the numerical simulation of sytems of particles submitted to inelastic collisions are studied in [9] by the second author.

We emphasize that in the previously mentioned works, the different numerical schemes (permitting to discretize (1.1)) are written (or can be written) in a multiconstraint case. The main difficulties consist in proving on the one hand the existence of solutions for (1.1) and on the other hand a convergence result for the associated numerical schemes for such multi-constrained problems. Concerning the uniqueness, we know from [26] and [1] that even with smooth data the uniqueness does not hold. The only positive results are proved in [27] for one-dimensional impact problems and in [1], in the context of analytic data. This critical question of uniqueness is not studied here.

\section{The framework}

In this work, we are interested in extending the previous work [10], in order to prove the existence of solutions and to get a convergence result of the scheme in the case of multiple time-dependent constraints. Moreover, we give applications in modeling inelastic collisions.

First of all, let us precise some notations. We write $W^{1, \infty}\left(I, \mathbb{R}^{d}\right)$ (respectively, $W^{1,1}\left(I, \mathbb{R}^{d}\right)$ ) for the Sobolev space of functions in $L^{\infty}\left(I, \mathbb{R}^{d}\right)$ (respectively, $\left.L^{1}\left(I, \mathbb{R}^{d}\right)\right)$ whose derivative is also in $L^{\infty}\left(I, \mathbb{R}^{d}\right)$ (respectively, $\left.L^{1}\left(I, \mathbb{R}^{d}\right)\right) . B V\left(I, \mathbb{R}^{d}\right)$ is the space of functions in $L^{\infty}\left(I, \mathbb{R}^{d}\right)$ with bounded variations on $I$. We define the 
dual space $\mathcal{M}(I):=\left(\mathcal{C}_{c}(I)\right)^{\prime}$ where $\mathcal{C}_{c}(I)$ is the space of continuous functions with compact support (corresponding to the set of Radon measure due to Riesz theorem). We set $\mathcal{M}_{+}(I)$ for the subset of positive measures.

We consider second-order differential inclusions involving a set-valued map $Q:[0, T] \rightrightarrows \mathbb{R}^{d}$ satisfying that for every $t \in[0, T], Q(t)$ is the intersection of complements of smooth convex sets. Let us first specify the set-valued map $Q$. This general framework has already been described by Venel in [30] for first-order differential inclusions (fitting into the so-called sweeping process theory) and in [2] for a stochastic perturbation of such problems.

For $i \in\{1, \ldots, p\}$, let $g_{i}:[0, T] \times \mathbb{R}^{d} \rightarrow \mathbb{R}$ be a convex function with respect to the second variable. For every $t \in[0, T]$, we introduce the sets $Q_{i}(t)$ defined by:

$$
Q_{i}(t):=\left\{q \in \mathbb{R}^{d}, g_{i}(t, q) \geq 0\right\},
$$

and the feasible set $Q(t)$ (supposed to be nonempty)

$$
Q(t):=\bigcap_{i=1}^{p} Q_{i}(t) .
$$

We denote by $I=[0, T]$ the time interval. The considered problem is the following: we are looking for a solution $q \in W^{1, \infty}\left(I, \mathbb{R}^{d}\right), \dot{q} \in B V\left(I, \mathbb{R}^{d}\right)$ such that

$$
\left\{\begin{array}{l}
\forall t \in I, \quad q(t) \in Q(t) \\
\frac{d^{2} q}{d t^{2}}+N(Q(\cdot), q(\cdot)) \ni f(\cdot, q(\cdot)), \\
\forall t \in I, \quad \dot{q}\left(t^{+}\right)=P_{\mathcal{C}_{t, q(t)}} \dot{q}\left(t^{-}\right) \\
q(0)=q_{0} \in \operatorname{int}[Q(0)], \\
\dot{q}(0)=u_{0},
\end{array}\right.
$$

where $N(Q(t), q(t))$ is the proximal normal cone of $Q(t)$ at $q(t)$ and $\mathcal{C}_{t, q}$ is the set of admissible velocities:

$$
\mathcal{C}_{t, q}:=\left\{u, \partial_{t} g_{i}(t, q)+\left\langle\nabla_{q} g_{i}(t, q), u\right\rangle \geq 0 \text { if } g_{i}(t, q)=0\right\},
$$

which corresponds to (1.2) in our framework.

We have to make assumptions on the constraints $g_{i}$. First we require some regularity: we suppose that there exist $c>0$ and open sets $U_{i}(t) \supset Q_{i}(t)$ for all $t$ in $[0, T]$ verifying

$$
d_{H}\left(Q_{i}(t), \mathbb{R}^{d} \backslash U_{i}(t)\right)>c,
$$

where $d_{H}$ denotes the Hausdorff distance. Moreover, we assume that there exist constants $\alpha, \beta, M>0$ such that for all $t$ in $[0, T], g_{i}(t, \cdot)$ belongs to $C^{2}\left(U_{i}(t)\right)$ and satisfies

$$
\begin{gathered}
\forall q \in U_{i}(t), \quad \alpha \leq\left|\nabla_{q} g_{i}(t, q)\right| \leq \beta, \\
\forall q \in U_{i}(t), \quad\left|\partial_{t} g_{i}(t, q)\right| \leq \beta,
\end{gathered}
$$




$$
\begin{gathered}
\forall q \in U_{i}(t), \quad\left|\partial_{t} \nabla_{q} g_{i}(t, q)\right| \leq M, \\
\forall q \in U_{i}(t), \quad\left|\mathrm{D}_{q}^{2} g_{i}(t, q)\right| \leq M
\end{gathered}
$$

and

$$
\forall q \in U_{i}(t),\left|\partial_{t}^{2} g_{i}(t, q)\right| \leq M .
$$

In comparison with [30] and [2] where first-order differential inclusion are studied, we require the new and natural assumption (A5), due to the fact that we consider second-order differential inclusions.

Note that these assumptions can slightly be weakened. Indeed, the lower bound in (A1) is only required in a neighborhood of $q \in \partial Q(t)$. Moreover, we have assumed $C^{2}$-smoothness in (A4) and (A5) for the sake of simplicity, but we only need $C^{1+\epsilon}$ regularity.

Furthermore, we require a kind of independence for the active gradients. For all $t \in[0, T]$ and $q \in Q(t)$, we denote by $I(t, q)$ the active set at $q$

$$
I(t, q):=\left\{i \in\{1, \ldots, p\}, g_{i}(t, q)=0\right\},
$$

corresponding to the active constraints. For every $\rho>0$, we define the following set:

$$
I_{\rho}(t, q):=\left\{i \in\{1, \ldots, p\}, g_{i}(t, q) \leq \rho\right\} .
$$

We assume there exist $\gamma>0$ and $\rho>0$ such that for all $t \in[0, T]$,

$$
\forall q \in Q(t), \quad \forall \lambda_{i} \geq 0, \quad \sum_{i \in I_{\rho}(t, q)} \lambda_{i}\left|\nabla_{q} g_{i}(t, q)\right| \leq \gamma\left|\sum_{i \in I_{\rho}(t, q)} \lambda_{i} \nabla_{q} g_{i}(t, q)\right| .
$$

We will use the following weaker assumption too:

$$
\forall q \in Q(t), \quad \forall \lambda_{i} \geq 0, \quad \sum_{i \in I(t, q)} \lambda_{i}\left|\nabla_{q} g_{i}(t, q)\right| \leq \gamma\left|\sum_{i \in I(t, q)} \lambda_{i} \nabla_{q} g_{i}(t, q)\right| .
$$

Note that assumptions (A6) and $\left(\mathrm{A} 6^{\prime}\right)$ describe a kind of "positive linear independence" of the almost active gradients. In the time-independent case, $\left(\mathrm{A} 6^{\prime}\right)$ is lightly weaker than the linear independence assumption made in [22-24]. Assumption (A6 $6^{\prime}$ implies the "uniform prox-regularity" of the admissible set $Q(t)$, which is a weaker property than the convexity. Indeed, a set is said to be uniformly prox-regular when one can move a ball of constant radius on its boundary, this ball staying outside the considered set. This implies the fact that any point close enough to the set can be projected on it. In case of time-dependent constraints, one has to impose (A6) in order to control the dependence in time. This stronger hypothesis will allow us to prove that the map $t \rightarrow Q(t)$ is Lipschitz and to have the possibility to find "good directions" (see the fundamental Lemma 3.1). We emphasize that the notion of prox-regularity is very useful for the study of first-order differential inclusions (the so-called sweeping process) and naturally appears in this context. In the framework 
of second-order differential inclusions, this notion is less important. We refer to [3] for a general result about second-order differential inclusions (involving the notion of "admissible" sets which is related to the notion of "good directions" appearing in Lemma 3.1).

Under these assumptions, we have a characterization of the proximal normal cone $N(Q(t), \cdot)$.

Proposition 1.2. (Proposition 2.8 of [30]) In this framework, we know that for every $t \in I$, and every $q \in \partial Q(t)$,

$$
N(Q(t), q):=\left\{-\sum_{i \in I(t, q)} \lambda_{i} \nabla_{q} g_{i}(t, q), \lambda_{i} \geq 0\right\} .
$$

So our problem (1.6) can be written as follows: we are looking for solutions $q \in W^{1, \infty}\left(I, \mathbb{R}^{d}\right), \dot{q} \in B V\left(I, \mathbb{R}^{d}\right)$ and time-measures $\lambda_{i} \in \mathcal{M}_{+}(I)$ such that

$$
\left\{\begin{array}{l}
\forall t \in I, \quad q(t) \in Q(t) \\
\frac{d^{2} q}{d t^{2}}=f(\cdot, q(\cdot))+\sum_{i=1}^{p} \lambda_{i} \nabla_{q} g_{i}(\cdot, q(\cdot)), \\
\operatorname{supp}\left(\lambda_{i}\right) \subset\left\{t, g_{i}(t, q(t))=0\right\} \quad \text { for all } i \\
\forall t \in I, \quad \dot{q}\left(t^{+}\right)=P_{C_{t, q(t)}} \dot{q}\left(t^{-}\right) \\
q(0)=q_{0} \in \operatorname{int}[Q(0)], \\
\dot{q}(0)=u_{0} .
\end{array}\right.
$$

We denote by $\lambda=\left(\lambda_{1}, \ldots, \lambda_{p}\right) \in \mathbb{R}^{p}$ the vector of the Lagrange multipliers associated to these $p$ constraints.

As usual, we obtain the existence results for (1.10) by proving the convergence of a sequence of discretized solutions.

To do so, we extend the algorithm proposed by Maury in [10] for modeling inelastic collisions, to the case of abstract and time-dependent constraints. In [10], the convergence (up to a subsequence) is proved in the case of a single constraint. Here, we show that this convergence still holds in the multi-constraint case.

Let us describe the numerical scheme. Let $h=T / N$ be the time step. We denote by $q_{h}^{n} \in \mathbb{R}^{d}$ and $u_{h}^{n} \in \mathbb{R}^{d}$ the approximated solution and velocity at time $t_{h}^{n}=n h$ for $n \in\{0, \ldots, N\}$.

The discretization of the continuous constraints $\mathcal{C}_{t_{h}^{n}, q\left(t_{h}^{n}\right)}$ proposed in [10] corresponds to a first-order approximation of the constraints in the space variable: for $t \in I$ and $q \in U(t)$, we set

$$
K_{h}(t, q):=\left\{u, g_{i}(t, q)+h\left\langle\nabla_{q} g_{i}(t, q), u\right\rangle \geq 0\right\} .
$$

The reason why we do not expand the time variable in this discrete admissible set is that we will use a semi-implicit numerical scheme, with direct impliciting in time the set $K_{h}(t, q)$ (see (1.13)). 
The approximated solutions are built using the following schemes:

(1) Initialization:

$$
\left(q_{h}^{0}, u_{h}^{0}\right):=\left(q_{0}, u_{0}\right)
$$

(2) Time iterations: $q_{h}^{n}$ and $u_{h}^{n}$ are given. We define $f_{h}^{n}:=\frac{1}{h} \int_{t_{h}^{n}}^{t_{h}^{n+1}} f\left(s, q_{h}^{n}\right) d s$,

$$
u_{h}^{n+1}:=P_{K_{h}\left(t_{h}^{n+1}, q_{h}^{n}\right)}\left[u_{h}^{n}+h f_{h}^{n}\right]
$$

and

$$
q_{h}^{n+1}:=q_{h}^{n}+h u_{h}^{n+1},
$$

where $P_{C}$ is the Euclidean projection onto the set $C$. This algorithm is a "predictioncorrection algorithm": the predicted velocity $u_{h}^{n}+h f_{h}^{n}$, that may not be admissible, is projected onto the approximate set of admissible velocities.

Since the projection $P_{K_{h}\left(t_{h}^{n+1}, q_{h}^{n}\right)}$ consists in a constrained minimization problem, with a finite number of affine constraints, it involves Lagrange multipliers $\left(\lambda_{h}^{n+1}\right) \in \mathbb{R}^{p}$ corresponding to the $p$ constraints. It can be checked that we have a discrete counterpart of the momentum balance appearing in (1.10):

$$
\frac{u_{h}^{n+1}-u_{h}^{n}}{h}=f_{h}^{n}+\sum \lambda_{h, i}^{n+1} \nabla_{q} g_{i}\left(t_{h}^{n+1}, q_{h}^{n}\right)
$$

with $\lambda_{h, i}^{n+1} \geq 0$ and $\lambda_{h, i}^{n+1}=0$ when $g_{i}\left(t_{h}^{n+1}, q_{h}^{n}\right)+h\left\langle\nabla_{q} g_{i}\left(t_{h}^{n+1}, q_{h}^{n}\right), u_{h}^{n+1}\right\rangle>0$.

In [10], the scheme is shown to be stable, robust and to present a good behavior for large time-steps. That is why, we are interested in continuing its numerical analysis in the multi-constraint case, with proposing some extensions like the timedependence of the constraints.

\section{Results}

We recall that $I=[0, T]$ is the time interval and $h$ is the constant time step $\left(t_{h}^{n}=n h\right.$ for $n=0, \ldots, N)$. We denote by $q_{h}$ the piecewise affine function with $q_{h}\left(t_{h}^{n}\right)=q_{h}^{n}$.

We denote by $u_{h}$ the derivative of $q_{h}$, piecewise constant equal to $u_{h}^{n+1}$ on ]$t_{h}^{n}, t_{h}^{n+1}\left[\right.$. Finally, we define $\lambda_{h}$, piecewise constant equal to $\lambda_{h}^{n+1}$ on $] t_{h}^{n}, t_{h}^{n+1}[$.

The convergence theorem is the following.

Theorem 1.3. Let $\left(q_{h}, u_{h}, \lambda_{h}\right)$ be the sequence of solutions constructed from the scheme (1.12)-(1.15) and suppose that $f: I \times \mathbb{R}^{d} \rightarrow \mathbb{R}^{d}$ is a measurable map satisfying:

$$
\begin{aligned}
& \exists K_{L}>0, \quad \forall t \in I, \quad \forall q, \tilde{q} \in U(t), \quad|f(t, q)-f(t, \tilde{q})| \leq K_{L}|q-\tilde{q}|, \\
& \exists F \in L^{1}(I), \quad \forall t \in I, \quad \forall q \in U(t), \quad|f(t, q)| \leq F(t) .
\end{aligned}
$$

Then, when $h$ goes to zero, there exist subsequences, still denoted by $\left(q_{h}\right)_{h},\left(u_{h}\right)_{h},\left(\lambda_{h}\right)_{h}$, and

$$
(q, u, \lambda) \in W^{1, \infty}\left(I, \mathbb{R}^{d}\right) \times B V\left(I, \mathbb{R}^{d}\right) \times \mathcal{M}_{+}(I)^{p}
$$


such that

$$
\begin{array}{ll}
u_{h} \longrightarrow u & \text { in } L^{1}\left(I, \mathbb{R}^{d}\right), \\
q_{h} \longrightarrow q & \text { in } W^{1,1}\left(I, \mathbb{R}^{d}\right) \quad \text { and } \quad L^{\infty}\left(I, \mathbb{R}^{d}\right) \quad \text { with } \dot{q}=u, \\
\lambda_{h} \stackrel{\star}{\longrightarrow} \quad \text { in } \mathcal{M}_{+}(I)^{p}, &
\end{array}
$$

where $(q, u, \lambda)$ is solution to (1.10) and so $(q, u)$ is a solution to (1.6).

We emphasize that, up to our knowledge, this result is the first one concerning such multi-constrained second-order differential inclusions with on the one hand time-dependent constraints and on the other hand a nonconvex and nonsmooth admissible set.

Remark 1.4. For time-independent constraints, Assumption $\left(\mathrm{A} 6^{\prime}\right)$ is required but Assumption (A6) is not necessary.

Remark 1.5. It is important to emphasize that Theorem 1.3 gives a global existence result since the time $T$ can be chosen independently with respect to the initial data. This improves the results in the literature (described in the introduction) which were local such that in case of time-dependent constraints, the time $T$ was depending on the initial data. Note that, in the case of nondepending on time, global results in a more regular setting were already proved in $[1,12,18,22,26]$.

The proof is quite long and technical. We refer the reader to [10] for a first proof dealing with the case of one $(p=1)$ time-independent constraint $g$. We will follow the same reasoning with some new arguments (appearing in [30]) in order to solve the difficulties raised by the multiple constraints and the timedependence. Section 2 is devoted to the outline and the main ideas of the proof. For the sake of readibility, the demonstrations of some technical propositions are postponed to Sec. 3. In Sec. 4, we describe an application to the modeling of inelastic collisions.

\section{Convergence Result}

This section is devoted to the proof of Theorem 1.3. It is divided into 7 steps and for readibility reasons we have postponed some technical proofs in the next section.

- Step 1. The scheme is well-defined and produces feasible configurations

Proposition 2.1. For a small enough parameter $h$, the scheme is well-defined. Moreover, the computed configurations are feasible:

$$
\forall h>0, \quad \forall n \in\{0, \ldots, N\}, \quad q_{h}\left(t_{h}^{n}\right) \in Q\left(t_{h}^{n}\right) .
$$


Proof. Let $h$ be smaller than $c / c_{0}$ where $c$ and $c_{0}$ are given in Lemma 2.2 (below stated) and Assumption (A0) respectively. By assuming that $q_{h}\left(t_{h}^{n}\right) \in$ $Q\left(t_{h}^{n}\right)$, we also deduce that $q_{h}\left(t_{h}^{n}\right) \in Q\left(t_{h}^{n+1}\right)+c_{0} h \bar{B}(0,1) \subset U\left(t_{h}^{n+1}\right)$. Then the gradient $\nabla_{q} g_{i}\left(t_{h}^{n+1}, q_{h}^{n}\right)$ is well-defined and so is the set $K_{h}\left(t_{h}^{n+1}, q_{h}^{n}\right)$. Step 2 of the scheme can be performed and due to the convexity of function $g_{i}\left(t_{h}^{n+1}, \cdot\right)$,

$$
u_{h}^{n+1} \in K_{h}\left(t_{h}^{n+1}, q_{h}^{n}\right) \Rightarrow q_{h}^{n+1} \in Q\left(t_{h}^{n+1}\right) .
$$

Then we conclude by iteration.

Lemma 2.2. The set-valued map $Q$ is Lipschitz continuous with a constant $c_{0}$, for the Hausdorff distance.

We refer the reader to Proposition 2.11 of [30] for a detailed proof of this result. For the intermediate times $t \in] t_{h}^{n}, t_{h}^{n+1}\left[\right.$, the point $q_{h}(t)$ may not belong to $Q(t)$. However, from Proposition 2.1 and Lemma 2.2, we have the following estimate:

$$
\forall h>0, \quad \forall t \in I, \quad d\left(q_{h}(t), Q(t)\right) \leq \max \left\{d\left(q_{h}^{n}, Q(t)\right), d\left(q_{h}^{n+1}, Q(t)\right)\right\} \leq c_{0} h .
$$

- Step 2. $u_{h}$ is bounded in $B V\left(I, \mathbb{R}^{d}\right)$

First, we check that the velocities are uniformly bounded (proved later in Sec. 3.1).

Proposition 2.3. The sequence of computed velocities $\left(u_{h}\right)_{h}$ is bounded in $L^{\infty}\left(I, \mathbb{R}^{d}\right)$. We set

$$
K:=\sup _{h}\left\|u_{h}\right\|_{L^{\infty}(I)}<\infty .
$$

Proposition 2.4. The sequence of computed velocities $\left(u_{h}\right)_{h}$ is bounded in $B V\left(I, \mathbb{R}^{d}\right)$.

Proof. Since $u_{h}^{0}=u_{0}$ and using Proposition 2.3, it suffices to show that $\left(u_{h}\right)_{h}$ has bounded variations on $I$. This has been proved for a single constraint in [10]. Unfortunately, this proof cannot be extended to the multi-constraint case. To obtain an estimate on the total variation, we use a similar technique to the one proposed in [6] and [8]. These ideas rest on the following property: all the cones $K_{h}\left(t_{h}^{n+1}, q_{h}^{n}\right)$ contain a ball of fixed radius with a bounded center, which describes the fact that the solid angles of the cones $N\left(Q(t), q_{h}(t)\right)$ are not too small. This property is proved by using a "good direction" (see Lemma 3.1) which permits to increase all the almost active constraints. The details of the proof are postponed to Sec. 3.1.

- Step 3. Extraction of convergent subsequences 
Proposition 2.4 directly implies the following convergence result:

Proposition 2.5. There exist $q$ in $W^{1, \infty}\left(I, \mathbb{R}^{d}\right)$ and $u$ in $B V\left(I, \mathbb{R}^{d}\right)$ such that, up to a subsequence,

$$
\begin{array}{ll}
u_{h} \underset{h \rightarrow 0}{\longrightarrow} u \quad \text { in } L^{1}\left(I, \mathbb{R}^{d}\right), \\
q_{h} \underset{h \rightarrow 0}{\longrightarrow} q \quad \text { in } W^{1,1}\left(I, \mathbb{R}^{d}\right) \quad \text { and } \quad L^{\infty}\left(I, \mathbb{R}^{d}\right) \quad \text { with } \dot{q}=u .
\end{array}
$$

Furthermore, (2.1) yields

$$
\forall t \in I, \quad q(t) \in Q(t)
$$

In addition, we show that the sequence of Lagrange multipliers converges:

Proposition 2.6. There exists $\lambda$ in $\mathcal{M}_{+}(I)^{p}$ such that, up to a subsequence,

$$
\lambda_{h} \stackrel{\star}{\longrightarrow} \lambda \text { in } \mathcal{M}_{+}(I)^{p} \text {. }
$$

Proof. From (1.15), we have

$$
\sum_{i} h \lambda_{h, i}^{n+1} \nabla_{q} g_{i}\left(t_{h}^{n+1}, q_{h}^{n}\right)=u_{h}^{n+1}-u_{h}^{n}-h f_{h}^{n}
$$

with $\lambda_{h, i}^{n+1} \geq 0$ and $\lambda_{h, i}^{n+1}=0$ when

$$
g_{i}\left(t_{h}^{n+1}, q_{h}^{n}\right)+h\left\langle\nabla_{q} g_{i}\left(t_{h}^{n+1}, q_{h}^{n}\right), u_{h}^{n+1}\right\rangle>0 .
$$

Remark that for a small enough parameter $h, g_{i}\left(t_{h}^{n+1}, q_{h}^{n}\right)+h\left\langle\nabla_{q} g_{i}\left(t_{h}^{n+1}, q_{h}^{n}\right), u_{h}^{n}+\right.$ $\left.h f_{h}^{n}\right\rangle \leq 0$ implies that $i \in I_{\rho}\left(t_{h}^{n+1}, q_{h}^{n}\right)$. Consequently, the reverse triangle inequality (Assumption $\left(\mathrm{A} 6^{\prime}\right)$ ) with (A1) and the Lipschitz regularity (Assumption (A3)) imply for a small enough parameter $h$

$$
h \sum_{i} \lambda_{h, i}^{n+1} \lesssim\left|u_{h}^{n+1}-u_{h}^{n}-h f_{h}^{n}\right| .
$$

Therefore, we obtain for this small enough parameter $h$

$$
\left\|\lambda_{h}\right\|_{L^{1}(I)} \lesssim \operatorname{Var}_{I}\left(u_{h}\right)+\|F\|_{L^{1}(I)} .
$$

Hence from Proposition 2.4 together with hypothesis $(1.17),\left(\lambda_{h}\right)_{h}$ is bounded in $L^{1}(I)$, which concludes the proof.

- Step 4. Momentum balance

As in Step 6 of Theorem 1 in [10], using Proposition 2.5 together with Proposition 2.6, we pass to the limit in the discrete momentum balance (1.15) to obtain

Proposition 2.7. The momentum balance is verified by the limits $u$ and $\lambda$ : in the sense of time-measure

$$
\dot{u}=f(\cdot, q(\cdot))+\sum_{i} \lambda_{i} \nabla_{q} g_{i}(\cdot, q(\cdot)) .
$$


Note that this equation has to be thought in terms of time-measure (see Remark 1.1):

$$
d u=f(\cdot) d t+\sum_{i} \nabla_{q} g_{i}(\cdot, q(\cdot)) \lambda_{i},
$$

where we denote by $d u$ the differential measure of the $B V$-function $u$.

- Step 5. Support of the measures $\lambda_{i}$

From the uniform convergence of $q_{h}$ and the Lipschitz regularity of $g_{i}$ (Assumptions (A1) and (A2)), it can be checked, as in Step 7 of Theorem 1 in [10], that

\section{Proposition 2.8.}

$$
\forall i, \operatorname{supp}\left(\lambda_{i}\right) \subset\left\{t, g_{i}(t, q(t))=0\right\} .
$$

Indeed (2.2) describes a similar property for the discretized multipliers. The uniform convergence allows us to go to the limit in (2.2) and to prove the previous proposition.

This property describes the fact that the measure $\lambda_{i}$ has a contribution only when the associated constraint $g_{i}$ is saturated.

- Step 6. Initial condition

As in Step 8 of Theorem 1 in [10], using again the uniform convergence of $q_{h}$ it can be shown that

$$
q(0)=q_{0} \quad \text { and } \quad u(0)=u_{0} .
$$

We emphasize that to prove this point, we use the property $q_{0} \in \operatorname{Int}[Q(0)]$. From this, it can be checked that for $t_{h}^{n}<s$ with $s$ a small enough parameter the desired velocity $u_{h}^{n}+h f_{h}^{n}$ still remains admissible and so we do not need to project. That allows us to deal with any initial velocity $u_{0} \in \mathbb{R}^{d}$. If $q_{0} \in \partial Q(0)$, this property still holds if we assume that the initial velocity is admissible: $u_{0} \in \mathcal{C}_{0, q_{0}}$. Else, we would get

$$
u^{+}(0)=P_{\mathcal{C}_{0, q_{0}}}\left(u_{0}\right)
$$

according to the next proposition.

- Step 7. Collision law

Finally, Theorem 1.3 will follow, provided that we check the collision law for the limits $u$ and $q$, which is given by the following proposition.

\section{Proposition 2.9.}

$$
\forall t_{0} \in I, \quad u^{+}\left(t_{0}\right)=P_{\mathcal{C}_{t_{0}, q\left(t_{0}\right)}}\left(u^{-}\left(t_{0}\right)\right)
$$

Proof. The idea is to let $h$ go to zero in the discrete collision law,

$$
u_{h}^{n+1}=P_{K_{h}\left(t_{h}^{n+1}, q_{h}^{n}\right)}\left[u_{h}^{n}+h f_{h}^{n}\right] .
$$

The main difficulty comes from the fact that the mapping $q \rightarrow K_{h}(t, q)$ is not Lipschitzian. The details of the proof are postponed to Sec. 3.2. 


\section{Auxiliary Results}

Before proving the technical Propositions 2.3, 2.4 and 2.9, we recall the following main lemma. This technical result is very important and all our proofs rest on this idea. It says that, for each time $t_{h}^{n}$, one can find a "good direction" increasing all the constraints which are almost active, the corresponding increase being independent of $n$.

Lemma 3.1. There exist constants $\delta, \kappa, \theta$ and $\tau>0$ such that for all $t \in I$, for all $q \in \partial Q(t)$ there exists a unit vector $v:=v(t, q)$ satisfying:

- for all $s \in[t-\tau, t+\tau], y \in Q(s) \cap B(q, \theta)$ and $i \in I_{\kappa \rho}(s, y)$,

$$
\left\langle\nabla_{q} g_{i}(s, y), v\right\rangle \geq \delta
$$

where $\rho$ is the constant defined in (A6).

This lemma is a consequence of the reverse triangle inequality (Assumption (A6)). We do not give the proof here and refer the reader to Lemma 2.10 in [30] for a detailed proof with $\tau=0$ and to Lemma 5.2 in [2] for a complete proof with some $\tau>0$. Indeed in the previously mentioned papers, a detailed construction of such "good directions" can be found.

\section{1. $B V$ estimate for $u_{h}$ (Propositions 2.3 and 2.4)}

We first prove a uniform bound of the computed velocities $u_{h}$ in $L^{\infty}(I)$.

Proof of Proposition 2.3. (1) For any $t \in I$ and $q \in Q(t)$, construction of a specific point $w \in \mathcal{C}_{t, q}$. From Lemma 3.1, there exists a "good direction": a unit vector $v$ satisfying,

$$
\forall i \in I_{l}(t, q), \quad\left\langle\nabla_{q} g_{i}(t, q), v\right\rangle \geq \delta,
$$

for some numerical constants $\delta, l>0$ nondepending on $t$ and $q$. For $k>0$, a large enough number, we claim that $k v$ belongs to $\mathcal{C}_{t, q}$. Indeed for $i \in I_{l}(t, q)(\supset I(t, q))$, with $k \geq(\beta+\delta) / \delta$, it comes

$$
\partial_{t} g_{i}(t, q)+\left\langle\nabla_{q} g_{i}(t, q), k v\right\rangle \geq \partial_{t} g_{i}(t, q)+k \delta \geq \delta>0,
$$

thanks to Assumption (A2).

Choosing $k=(\beta+\delta) / \delta$, we have built a point $w=k v$ belonging to $\mathcal{C}_{t, q}$ with $|w|=(\beta+\delta) / \delta$.

(2) This vector $w$ belongs to $K_{h}(s+h, \tilde{q})$ for $(s, \tilde{q})$ close to $(t, q)$ and $h$ small enough. Let fix a point $(t, q)$ (for example the initial condition $(t, q)=\left(0, q_{0}\right)$ ). From the previous point, we know that there exists a bounded admissible velocity $w \in \mathcal{C}_{t, q}$, which satisfies the stronger property (3.2). 
More precisely if $s \in I$ and $\tilde{q} \in Q(s)$ satisfy

$$
|s-t|+|\tilde{q}-q| \leq \min \{l /(2 \beta), \epsilon\}:=\nu
$$

with $\epsilon$ a small parameter verifying $2 \epsilon M(1+(\beta+\delta) / \delta) \leq \delta$ then $w \in K_{h}(s+h, \tilde{q})$. Indeed, from (3.3) we have

$$
I_{l / 2}(s, \tilde{q}) \subset I_{l}(t, q) .
$$

This, together with (3.2) and (3.3) gives, for all $i \in I_{l / 2}(s, \tilde{q})$

$$
\partial_{t} g_{i}(s, \tilde{q})+\left\langle\nabla_{q} g_{i}(s, \tilde{q}), w\right\rangle \geq \delta-\epsilon M(1+|w|) \geq \delta / 2 .
$$

Moreover, for the other indices $i \notin I_{l / 2}(s, \tilde{q})$ we have

$$
g_{i}(s, \tilde{q})+h\left[\partial_{t} g_{i}(s, \tilde{q})+\left\langle\nabla_{q} g_{i}(s, \tilde{q}), w\right\rangle\right] \geq \frac{l}{2}-h \beta(1+|w|) .
$$

Consequently, for $h$ small enough $\left(h \leq h_{0}:=l /(2 \beta(1+(\beta+\delta) / \delta)+\delta / 2)\right)$, we obtain

$$
\forall i, \quad g_{i}(s, \tilde{q})+h\left[\partial_{t} g_{i}(s, \tilde{q})+\left\langle\nabla_{q} g_{i}(s, \tilde{q}), w\right\rangle\right] \geq h \frac{\delta}{2},
$$

which, by a first-order expansion in time gives:

$$
\forall i, \quad g_{i}(s+h, \tilde{q})+h\left\langle\nabla_{q} g_{i}(s+h, \tilde{q}), w\right\rangle \geq h \frac{\delta}{2}+O_{h \rightarrow 0}\left(h^{2}\right) .
$$

We deduce that there exists $h_{1} \leq h_{0}$ such that, for $h \leq h_{1}$,

$$
\forall i, \quad g_{i}(s+h, \tilde{q})+h\left\langle\nabla_{q} g_{i}(s+h, \tilde{q}), w\right\rangle \geq 0,
$$

and consequently $w \in K_{h}(s+h, \tilde{q})$.

(3) Estimate on the velocities for small time intervals.

Let us fix $h \leq h_{1}$ (given in the previous point) and a small time interval $\left[t_{-}, t_{+}\right] \subset I$ of length

$$
\left|t_{+}-t_{-}\right| \leq \frac{\nu}{2\left(\left|u_{h}^{n_{0}}\right|+2 \frac{\beta+\delta}{\delta}+\int_{0}^{T} F(t) d t\right)},
$$

where $n_{0}$ is the smallest integer $n$ such that $t_{h}^{n} \geq t_{-}$. We assume that $t_{h}^{n_{0}} \in\left[t_{-}, t_{+}\right]$. We are looking for a bound on the velocity on this time interval. From the first two points, setting $(t, q)=\left(t_{h}^{n_{0}}, q_{h}^{n_{0}}\right)$, we have an admissible velocity $w \in \mathcal{C}_{t, q}$ such that for all $s \in I$ and $\tilde{q} \in Q(s)$ we have

$$
w \in K_{h}(s+h, \tilde{q})
$$

as soon as $|s-t|+|\tilde{q}-q| \leq \nu$. Since for $s=t_{h}^{n} \in\left[t_{-}, t_{+}\right],|s-t| \leq \nu / 2$, we deduce that for all integers $n$ such that $t_{h}^{n} \in\left[t_{-}, t_{+}\right]$, if

$$
\left|q_{h}^{n}-q_{h}^{n_{0}}\right| \leq \nu / 2
$$

then

$$
w \in K_{h}\left(t_{h}^{n+1}, q_{h}^{n}\right) .
$$


Considering such an integer $n$ satisfying (3.4), since $u_{h}^{n+1}$ is the Euclidean projection of $u_{h}^{n}+h f_{h}^{n}$ on the convex set $K_{h}\left(t_{h}^{n+1}, q_{h}^{n}\right)$ (containing the point $w$ ), we deduce that

$$
\left|u_{h}^{n+1}-w\right| \leq\left|u_{h}^{n}+h f_{h}^{n}-w\right|
$$

which implies

$$
\left|u_{h}^{n+1}-w\right| \leq\left|u_{h}^{n}-w\right|+\int_{t_{h}^{n}}^{t_{h}^{n+1}} F(t) d t .
$$

We set $m$ the smallest integer (bigger than $n_{0}$ ) such that $m+1$ does not satisfy (3.4) or $t_{h}^{m+1} \notin\left[t_{-}, t_{+}\right]$. By summing these inequalities from $n=n_{0}$ to $n=p-1$ with $n_{0} \leq p \leq m$, we get

$$
\forall p \in\left[n_{0}, m\right], \quad\left|u_{h}^{p}-w\right| \leq\left|u_{h}^{n_{0}}-w\right|+\int_{0}^{T} F(t) d t .
$$

Finally, it becomes

$$
\sup _{n_{0} \leq p \leq m}\left|u_{h}^{p}\right| \leq\left|u_{h}^{n_{0}}\right|+2 \frac{\beta+\delta}{\delta}+\int_{0}^{T} F(t) d t .
$$

By integrating in time, we deduce

$$
\left|q_{h}^{m+1}-q_{h}^{n_{0}}\right| \leq\left(\left|u_{h}^{n_{0}}\right|+2 \frac{\beta+\delta}{\delta}+\int_{0}^{T} F(t) d t\right)\left|t_{+}-t_{-}\right| \leq \nu / 2
$$

by the assumption on the length of the time interval. As a consequence, we get that $n=m+1$ satisfies (3.4) which by definition of $m$, yields $t_{h}^{m} \leq t_{+}<t_{h}^{m+1}$. Hence, from (3.5), we have

$$
\sup _{t_{-} \leq t_{h}^{n} \leq t_{+}}\left|u_{h}^{n}\right| \leq\left|u_{h}^{n_{0}}\right|+2 \frac{\beta+\delta}{\delta}+\int_{0}^{T} F(t) d t .
$$

(4) End of the proof.

The parameter $h<h_{1}$ being fixed, we are now looking for a bound on $u_{h}$ on the whole time interval $I=[0, T]$. Let us start with $t_{-}=t(0):=0$. From the previous point we know that with

$$
t_{+}=t(1):=\min \left\{\frac{\nu}{2\left(\left|u_{0}\right|+2 \frac{\beta+\delta}{\delta}+\int_{0}^{T} F(t) d t\right)}, T\right\}
$$

we have

$$
\sup _{0 \leq t_{h}^{n} \leq t(1)}\left|u_{h}\left(t_{h}^{n}\right)\right| \leq\left|u_{0}\right|+2 \frac{\beta+\delta}{\delta}+\int_{0}^{T} F(t) d t
$$


Then, let us assume that there exists $n_{1}$ such that $t(0)<t_{h}^{n_{1}} \leq t(1)<t_{h}^{n_{1}+1}$. We have $0 \leq \delta_{1}:=t(1)-t_{h}^{n_{1}}<h$. In that case, we set $t_{-}=t_{h}^{n_{1}}$ and

$$
\begin{aligned}
t_{+}=t(2) & :=\min \left\{t^{n_{1}}+\frac{\nu}{2\left(\left|u_{0}\right|+4 \frac{\beta+\delta}{\delta}+2 \int_{0}^{T} F(t) d t\right)}, T\right\} \\
& =\min \left\{t(1)-\delta_{1}+\frac{\nu}{2\left(\left|u_{0}\right|+4 \frac{\beta+\delta}{\delta}+2 \int_{0}^{T} F(t) d t\right)}, T\right\} .
\end{aligned}
$$

From the previous point, we deduce that

$$
\sup _{t(1) \leq t_{h}^{n} \leq t(2)}\left|u_{h}\left(t_{h}^{n}\right)\right| \leq \sup _{t^{n_{1}} \leq t_{h}^{n} \leq t(2)}\left|u_{h}\left(t_{h}^{n}\right)\right| \leq\left|u_{0}\right|+4 \frac{\beta+\delta}{\delta}+2 \int_{0}^{T} F(t) d t
$$

and so

$$
\sup _{0 \leq t_{h}^{n} \leq t(2)}\left|u_{h}\left(t_{h}^{n}\right)\right| \leq\left|u_{0}\right|+4 \frac{\beta+\delta}{\delta}+2 \int_{0}^{T} F(t) d t .
$$

By iterating this reasoning, for any integer $k \geq 1$ we set

$$
\begin{aligned}
t(k) & :=\min \left\{t(k-1)-\delta_{k-1}+\frac{\nu}{2\left(\left|u_{0}\right|+2 k \frac{\beta+\delta}{\delta}+2 k \int_{0}^{T} F(t) d t\right)}, T\right\} \\
& =\min \left\{-\sum_{i=1}^{k-1} \delta_{i}+\sum_{i=1}^{k} \frac{\nu}{2\left(\left|u_{0}\right|+2 i \frac{\beta+\delta}{\delta}+2 i \int_{0}^{T} F(t) d t\right)}, T\right\},
\end{aligned}
$$

where $\delta_{k}<h$ for all $k$. This construction of $t(k)$ can be made while there exists $n_{k}$ such that $t(k-2)<t_{h}^{n_{k-1}} \leq t(k-1)<t_{h}^{n_{k-1}+1}$. That is, while $t(k-1)-t(k-2)>h$. This condition will be verified as long as

$$
-\delta_{k-2}+\frac{\nu}{2\left(\left|u_{0}\right|+2(k-1) \frac{\beta+\delta}{\delta}+2(k-1) \int_{0}^{T} F(t) d t\right)}>h .
$$

Therefore, using the fact that $0 \leq \delta_{k-2}<h$, we see that we can construct $t(k)$ for $k<N$ verifying

$$
\frac{\nu}{2\left(\left|u_{0}\right|+2(k-1) \frac{\beta+\delta}{\delta}+2(k-1) \int_{0}^{T} F(t) d t\right)}>2 h,
$$

which is equivalent to

$$
k<k_{0}(h):=1+\left(\frac{\nu}{8 h}-\frac{\left|u_{0}\right|}{2}\right)\left(\frac{\beta+\delta}{\delta}+\int_{0}^{T} F(t) d t\right)^{-1} .
$$


Consequently, we know that the velocities can be bounded on $\left[0, t\left(k_{0}(h)\right)\right]$ where

$$
t\left(k_{0}(h)\right)=\min \left\{-\sum_{i=1}^{k_{0}(h)-1} \delta_{i}+\sum_{i=1}^{k_{0}(h)} \frac{\nu}{2\left(\left|u_{0}\right|+2 i \frac{\beta+1}{\delta}+2 i \int_{0}^{T} F(t) d t\right)}, T\right\} .
$$

Now, using the fact that $k_{0}(h)$ goes to infinity when $h$ goes to zero, that the harmonic serie diverges and that (3.6) yields

$$
\left|\sum_{i=1}^{k_{0}(h)-1} \delta_{i}\right| \leq h k_{0}(h) \leq C,
$$

we see that $t\left(k_{0}(h)\right)$ is equal to $T$ for $h$ small enough. Therefore, there exists $h_{2}<h_{1}$ such that, for $h<h_{2}, T=t\left(k_{0}\left(h_{2}\right)\right)=t\left(k_{0}(h)\right)$. Finally, we see that, for $h<h_{2}$, $t(k)$ can be constructed until $k=k_{0}\left(h_{2}\right)$ and $u_{h}$ can be bounded as follows:

$$
\sup _{h \leq h_{2}} \sup _{0 \leq t_{h}^{n} \leq T}\left|u_{h}\left(t_{h}^{n}\right)\right| \leq\left|u_{0}\right|+2 k_{0}\left(h_{2}\right) \frac{\beta+\delta}{\delta}+2 k_{0}\left(h_{2}\right) \int_{0}^{T} F(t) d t
$$

which concludes the proof of the existence of a uniform bound in $L^{\infty}$ for the velocities $u_{h}$.

To prove Proposition 2.4, it suffices now to show that the sequence $\left(u_{h}\right)_{h}$ has bounded variation.

Theorem 3.2. The sequence $\left(u_{h}\right)_{h}$ has a bounded variation on $I$.

Proof. In order to study the variation of $u_{h}$ on $I$, we split $I$ into smallest intervals. We define $\left(s_{j}\right)_{j}$ for $j$ from 0 to $P$ such that:

$$
\left\{\begin{array}{l}
s_{0}=0, s_{P}=T \\
\left|s_{j+1}-s_{j}\right|=\frac{1}{2} \min \left\{\tau, \frac{\theta}{K}\right\}, \quad \text { for } j=0, \ldots, P-2, \\
\left|s_{P}-s_{P-1}\right| \leq \frac{1}{2} \min \left\{\tau, \frac{\theta}{K}\right\},
\end{array}\right.
$$

where $\tau$ and $\theta$ are given by Lemma 3.1 and $K$ is the bound on $\left\|u_{h}\right\|_{L^{\infty}(I)}$ (see Proposition 2.3). All these constants do not depend on $h$ and such a construction gives

$$
P=\left[\frac{2 T}{\min \left\{\tau, \frac{\theta}{K}\right\}}\right]+1
$$

which is independent of $h$. Then, for all $h$, we define $n_{h}^{j}$ for $j$ from 0 to $P-1$ as the first time step strictly greater than $s_{j}$ :

$$
t_{h}^{n_{h}^{j}-1} \leq s_{j}<t_{h}^{n_{h}^{j}}
$$

and $n_{h}^{P}$ is set equal to $N\left(t_{h}^{N}=t_{h}^{n_{h}^{P}}=T\right)$. 
In the following, we suppose $h<\min \left\{\left|s_{j+1}-s_{j}\right|\right\} / 2$. Doing so, we obtain a strictly increasing sequence of $\left(t_{h}^{n_{h}^{j}}\right)_{j}$ with

$$
\left|t_{h}^{n_{h}^{j}}-t_{h}^{n_{h}^{j-1}}\right| \leq \min \left\{\tau, \frac{\theta}{K}\right\} .
$$

The variation of $u_{h}$ on $I$ can be written as follows:

$$
\operatorname{Var}_{I}\left(u_{h}\right)=\sum_{n=0}^{N-1}\left|u_{h}^{n+1}-u_{h}^{n}\right|=\sum_{j=0}^{P-1} \operatorname{Var}_{j} u_{h}
$$

where

$$
\operatorname{Var}_{j}\left(u_{h}\right):=\sum_{n=n_{h}^{j}}^{n_{h}^{j+1}-1}\left|u_{h}^{n+1}-u_{h}^{n}\right|
$$

corresponds to the variation on $\left[t_{h}^{n_{h}^{j}}, t_{h}^{n_{h}^{j+1}}[\right.$. To study these terms, we recall that

$$
u_{h}^{n+1}=P_{K_{h}\left(t_{h}^{n+1}, q_{h}^{n}\right)}\left[u_{h}^{n}+h f^{n}\right]
$$

by construction and state the following lemma:

Lemma 3.3. There exist $\eta>0$ and uniformly bounded vectors $y^{n_{h}^{j}}$ such that, for all small enough $h$, for all $j=0, \ldots, P$ and $n \in\left[n_{h}^{j}, n_{h}^{j+1}[\right.$, we have

$$
x_{1}=P_{K_{h}\left(t_{h}^{n+1}, q_{h}^{n}\right)}\left[x_{0}\right] \Longrightarrow\left|x_{1}-x_{0}\right| \leq \frac{1}{2 \eta}\left(\left|x_{0}-y^{n_{h}^{j}}\right|^{2}-\left|x_{1}-y^{n_{h}^{j}}\right|^{2}\right) .
$$

Proof. The outline of the proof is the following: first, we prove that there exist unit vectors $v^{n_{h}^{j}}$ such that

$$
n \in\left[n_{h}^{j}, n_{h}^{j+1}\left[\Longrightarrow \bar{B}\left(\frac{2 \beta K}{\delta} v^{n_{h}^{j}}, \eta\right) \subset K_{h}\left(t_{h}^{n+1}, q_{h}^{n}\right) \quad \text { with } \eta:=\frac{K}{2},\right.\right.
$$

where $K$ is a bound on $\left\|u_{h}\right\|_{L^{\infty}(I)}$ (see Proposition 2.3). Then, we conclude using similar arguments to the ones exposed in $[6,8]$.

Step 1. From Lemma 3.1 with $t=t_{h}^{n_{h}^{j}}$ and $q=q_{h}^{n_{h}^{j}}$, we have a unit "good direction" written $v^{n_{h}^{j}}$. Let $n$ belong to $\left[n_{h}^{j}, n_{h}^{j+1}\right.$ [. From Proposition 2.1, we know that $q_{h}^{n+1}$ belongs to $Q\left(t_{h}^{n+1}\right)$. Moreover, (3.9) gives $\left|t_{h}^{n+1}-t_{h}^{n_{h}^{j}}\right| \leq \tau$ and $\left|q_{h}^{n+1}-q_{h}^{n_{h}^{j}}\right| \leq \theta$. Consequently, Lemma 3.1 gives

$$
\forall i \in I_{\kappa \rho}\left(t_{h}^{n+1}, q_{h}^{n+1}\right), \quad\left\langle\nabla_{q} g_{i}\left(t_{h}^{n+1}, q_{h}^{n+1}\right), v^{n_{h}^{j}}\right\rangle \geq \delta .
$$

We deduce that for all index $i \in\{1, \ldots, p\}$ and a small enough parameter $h$ :

$$
g_{i}\left(t_{h}^{n+1}, q_{h}^{n}\right)+\frac{2 \beta K}{\delta} h\left\langle\nabla_{q} g_{i}\left(t_{h}^{n+1}, q_{h}^{n+1}\right), v^{n_{h}^{j}}\right\rangle \geq-h \beta K+2 h \beta K=h \beta K .
$$


Indeed, we write

$$
\begin{aligned}
g_{i}\left(t_{h}^{n+1}, q_{h}^{n}\right)+\frac{2 \beta K}{\delta} h\left\langle\nabla_{q} g_{i}\left(t_{h}^{n+1}, q_{h}^{n+1}\right), v^{n_{h}^{j}}\right\rangle \\
=\left[g_{i}\left(t_{h}^{n+1}, q_{h}^{n}\right)-g_{i}\left(t_{h}^{n+1}, q_{h}^{n+1}\right)\right] \\
\quad+\left[g_{i}\left(t_{h}^{n+1}, q_{h}^{n+1}\right)+\frac{2 \beta K}{\delta} h\left\langle\nabla_{q} g_{i}\left(t_{h}^{n+1}, q_{h}^{n+1}\right), v^{n_{h}^{j}}\right\rangle\right] .
\end{aligned}
$$

The first term can be estimated using (A1) and the bound $K$ on $\left\|u_{h}\right\|_{L^{\infty}(I)}$. In order to estimate the second term, if $i \in I_{\kappa \rho}\left(t_{h}^{n+1}, q_{h}^{n+1}\right)$, we use (3.12) together with the fact that $g_{i}\left(t_{h}^{n+1}, q_{h}^{n+1}\right) \geq 0$, which gives the required bound. In the case $i \notin I_{\kappa \rho}\left(t_{h}^{n+1}, q_{h}^{n+1}\right)$, we use $g_{i}\left(t_{h}^{n+1}, q_{h}^{n+1}\right) \geq \kappa \rho$ and (A1), which also gives the required bound for $h$ small enough.

Finally, (3.13) together with assumption (A1) implies that for all $v \in$ $\bar{B}\left(\frac{2 \beta K}{\delta} v^{n_{h}^{j}}, K / 2\right)$

$$
g_{i}\left(t_{h}^{n+1}, q_{h}^{n}\right)+h\left\langle\nabla_{q} g_{i}\left(t_{h}^{n+1}, q_{h}^{n}\right), v\right\rangle \geq h \beta K-\frac{h \beta K}{2}=\frac{h \beta K}{2} \geq 0,
$$

which proves (3.11).

Step 2. Let $n$ belong to $\left[n_{h}^{j}, n_{h}^{j+1}[\right.$. We define

$$
z^{n_{h}^{j}}:=y^{n_{h}^{j}}+\eta \frac{x_{0}-x_{1}}{\left|x_{0}-x_{1}\right|} \quad \text { where } y^{n_{h}^{j}}:=\frac{2 \beta K}{\delta} v^{n_{h}^{j}} .
$$

(Here we assume $x_{0} \neq x_{1}$, else the desired result is obvious.) From the previous step we have

$$
z^{n_{h}^{j}} \in \bar{B}\left(\frac{2 \beta K}{\delta} v^{n_{h}^{j}}, \eta\right) \subset K_{h}\left(t_{h}^{n+1}, q_{h}^{n}\right) .
$$

The point $x_{1}$ being the projection of $x_{0}$ onto the closed convex set $K_{h}\left(t_{h}^{n+1}, q_{h}^{n}\right)$, we get

$$
\left\langle x_{0}-x_{1}, z^{n_{h}^{j}}-x_{1}\right\rangle \leq 0
$$

From this we have

$$
\begin{aligned}
\left|x_{0}-y^{n_{h}^{j}}\right|^{2}= & \left|x_{1}-y^{n_{h}^{j}}\right|^{2}+\left|x_{0}-x_{1}\right|^{2} \\
& +2\left\langle z^{n_{h}^{j}}-y^{n_{h}^{j}}, x_{0}-x_{1}\right\rangle+2\left\langle x_{1}-z^{n_{h}^{j}}, x_{0}-x_{1}\right\rangle \\
\geq & \left|x_{1}-y^{n_{h}^{j}}\right|^{2}+2\left\langle z^{n_{h}^{j}}-y^{n_{h}^{j}}, x_{0}-x_{1}\right\rangle \\
\geq & \left|x_{1}-y^{n_{h}^{j}}\right|^{2}+2 \eta\left|x_{0}-x_{1}\right| .
\end{aligned}
$$

This, together with the fact that the vectors $y^{n_{h}^{j}}$ are uniformly bounded by $\frac{2 \beta K}{\delta}$, ends the proof of Lemma 3.3. 
We now come back to the proof of Theorem 3.2. For $n$ in $\left[n_{h}^{j}, n_{h}^{j+1}[\right.$, using (3.10) and the previous lemma ( $w i t h x_{0}=u_{h}^{n}+h f_{h}^{n}$ and $x_{1}=u_{h}^{n+1}$ ), it becomes

$$
\begin{aligned}
\left|u_{h}^{n+1}-u_{h}^{n}-h f_{h}^{n}\right| \leq & \frac{1}{2 \eta}\left(\left|x_{0}-y^{n_{h}^{j}}\right|^{2}-\left|x_{1}-y^{n_{h}^{j}}\right|^{2}\right) \\
\leq & \frac{1}{2 \eta}\left(\left|u_{h}^{n}+h f_{h}^{n}-y^{n_{h}^{j}}\right|^{2}-\left|u_{h}^{n+1}-y^{n_{h}^{j}}\right|^{2}\right) \\
\leq & \frac{1}{2 \eta}\left(\left|u_{h}^{n}-y^{n_{h}^{j}}\right|^{2}-\left|u_{h}^{n+1}-y^{n_{h}^{j}}\right|^{2}\right) \\
& +\frac{1}{2 \eta}\left|h f_{h}^{n}\right|^{2}+\frac{1}{\eta}\left|h f_{h}^{n}\right|\left|u_{h}^{n}-y^{n_{h}^{j}}\right| \\
\leq & \frac{1}{2 \eta}\left(\left|u_{h}^{n}-y^{n_{h}^{j}}\right|^{2}-\left|u_{h}^{n+1}-y^{n_{h}^{j}}\right|^{2}\right) \\
& +\frac{1}{2 \eta}\left|h f_{h}^{n}\right|^{2}+\frac{1}{\eta}\left|h f_{h}^{n}\right|(K+L),
\end{aligned}
$$

where $L:=2 \beta K / \delta$ (see Proposition 2.3 for the definition of $K$ ). By summing up these terms for $n$ from $n_{h}^{j}$ to $n_{h}^{j+1}-1$ we get

$$
\begin{aligned}
\operatorname{Var}_{j}\left(u_{h}\right)= & \sum_{n=n_{h}^{j}}^{n_{h}^{j+1}-1}\left|u_{h}^{n+1}-u_{h}^{n}\right| \leq \frac{1}{2 \eta}\left(\left|u_{h}^{n_{h}^{j}}-y^{n_{h}^{j}}\right|^{2}-\left|u_{h}^{n_{h}^{j+1}}-y^{n_{h}^{j}}\right|^{2}\right) \\
& +\sum_{n=n_{h}^{j}}^{n_{h}^{j+1}-1} \frac{1}{2 \eta}\left|h f_{h}^{n}\right|^{2}+\frac{1}{\eta}(K+L+\eta) \sum_{n=n_{h}^{j}}^{n_{h}^{j+1}-1}\left|h f_{h}^{n}\right|
\end{aligned}
$$

and finally

$$
\begin{aligned}
\operatorname{Var}\left(u_{h}\right)=\sum_{j=0}^{P-1} \operatorname{Var}_{j}\left(u_{h}\right) \leq & \frac{1}{2 \eta} \sum_{j=0}^{P-1}\left(\left|u_{h}^{n_{h}^{j}}-y^{n_{h}^{j}}\right|^{2}-\left|u_{h}^{n_{h}^{j+1}}-y^{n_{h}^{j}}\right|^{2}\right)+\frac{1}{2 \eta}\|F\|_{L^{1}(I)}^{2} \\
& +\frac{1}{\eta}(K+L+\eta)\|F\|_{L^{1}(I)} \\
\leq & \frac{1}{\eta}(K+L)^{2} P+\frac{1}{2 \eta}\|F\|_{L^{1}(I)}^{2}+\frac{1}{\eta}(K+L+\eta)\|F\|_{L^{1}(I)} .
\end{aligned}
$$

This completes the proof of Theorem 3.2, since $P$ does not depend on $h$ from (3.8).

\subsection{Collision law for the limits $u$ and $q$ (Proposition 2.9)}

This subsection is devoted to the proof of Proposition 2.9, recalled in the following theorem:

Theorem 3.4. Let $t_{0} \in I$ be fixed. The limit function $u$ verifies:

$$
u^{+}\left(t_{0}\right)=P_{\mathcal{C}_{t_{0}, q\left(t_{0}\right)}}\left(u^{-}\left(t_{0}\right)\right)
$$


Note that, from Proposition 2.5, $u \in B V(I)$, so that $u^{-}\left(t_{0}\right)$ and the $u^{+}\left(t_{0}\right)$ limits are well-defined.

The proof is quite technical so for an easy reference, we remember the definitions of the sets $\mathcal{C}_{t, q}$ (given in (1.7)):

$$
\mathcal{C}_{t, q}:=\left\{u, \partial_{t} g_{i}(t, q)+\left\langle\nabla_{q} g_{i}(t, q), u\right\rangle \geq 0, \text { if } g_{i}(t, q)=0\right\}
$$

and $K_{h}(t, q)$ (given in $(1.11)$ ):

$$
K_{h}(t, q):=\left\{u, g_{i}(t, q)+h\left\langle\nabla_{q} g_{i}(t, q), u\right\rangle \geq 0\right\} .
$$

Moreover, we recall that

$$
K:=\sup _{h}\left\|u_{h}\right\|_{L^{\infty}(I)}<\infty .
$$

The desired property

$$
u^{+}\left(t_{0}\right)=P_{\mathcal{C}_{t_{0}, q\left(t_{0}\right)}}\left(u^{-}\left(t_{0}\right)\right)
$$

can be seen as the limit (for $h$ going to 0 ) of the "discretized property"

$$
u_{h}^{n+1}=P_{K_{h}\left(t_{h}^{n+1}, q_{h}^{n}\right)}\left[u_{h}^{n}+h f^{n}\right] .
$$

Proof. First we claim that

$$
u^{+}\left(t_{0}\right) \in C_{t_{0}, q\left(t_{0}\right)}
$$

To verify this property, let us consider an index $i$ such that $g_{i}\left(t_{0}, q\left(t_{0}\right)\right)=0$. Then a first-order expansion gives:

$$
g_{i}\left(t_{0}+\epsilon, q\left(t_{0}+\epsilon\right)\right)=\epsilon\left[\partial_{t} g_{i}\left(t_{0}, q\left(t_{0}\right)\right)+\left\langle u^{+}\left(t_{0}\right), \nabla_{q} g_{i}\left(t_{0}, q\left(t_{0}\right)\right)\right\rangle\right]+o_{\epsilon \rightarrow 0}(\epsilon) .
$$

The feasibility of $q\left(t_{0}+\epsilon\right)$ (see Proposition 2.5) yields

$$
\partial_{t} g_{i}\left(t_{0}, q\left(t_{0}\right)\right)+\left\langle u^{+}\left(t_{0}\right), \nabla_{q} g_{i}\left(t_{0}, q\left(t_{0}\right)\right)\right\rangle \geq 0
$$

which corresponds to $(3.16)$.

Let us now come back to the proof of (3.14). As we just proved $u^{+}\left(t_{0}\right) \in \mathcal{C}_{t_{0}, q\left(t_{0}\right)}$ and since $\mathcal{C}_{t_{0}, q\left(t_{0}\right)}$ is a convex set, (3.14) is equivalent to

$$
\forall w \in \mathcal{C}_{t_{0}, q\left(t_{0}\right)}, \quad\left\langle u^{-}\left(t_{0}\right)-u^{+}\left(t_{0}\right), w-u^{+}\left(t_{0}\right)\right\rangle \leq 0 .
$$

So, in the following, let us choose $w \in \mathcal{C}_{t_{0}, q\left(t_{0}\right)}$. To prove (3.17), we construct a family of points $w_{\nu}$ for $\nu>0$ such that $w_{\nu}$ tends to $w$ when $\nu$ goes to zero and satisfies $w_{\nu} \in K_{h}(t+h, q)$ for $h$ sufficiently small and $(t, q)$ close to $\left(t_{0}, q\left(t_{0}\right)\right)$. Then, for each $\nu$, we go to the limit on $h, t$ and $q$ to show that $\left\langle u^{-}\left(t_{0}\right)-u^{+}\left(t_{0}\right), w_{\nu}-u^{+}\left(t_{0}\right)\right\rangle \leq 0$ and finally, we make $\nu$ go to zero to conclude. 
Step 1. From Lemma 3.1, there exists a neighborhood $U \subset I \times \mathbb{R}^{d}$ around $\left(t_{0}, q\left(t_{0}\right)\right)$ and $v \in \mathbb{R}^{d}$ such that for all $t \in I$ and $q \in Q(t)$

$$
(t, q) \in U \Rightarrow \forall i \in I_{\kappa \rho}(t, q),\left\langle\nabla_{q} g_{i}(t, q), v\right\rangle \geq \delta,
$$

with a numerical constant $\delta>0$. For $\nu>0$, we consider the point $w_{\nu}:=w+\nu v$ with $\nu>0$. For all $i \in I_{\kappa \rho}(t, q) \cap I\left(t_{0}, q\left(t_{0}\right)\right)$, (3.18) together with $w \in \mathcal{C}_{t_{0}, q\left(t_{0}\right)}$ gives

$$
\begin{aligned}
\partial_{t} g_{i}\left(t_{0}, q\left(t_{0}\right)\right)+\left\langle\nabla_{q} g_{i}(t, q), w_{\nu}\right\rangle & =\partial_{t} g_{i}\left(t_{0}, q\left(t_{0}\right)\right)+\left\langle\nabla_{q} g_{i}(t, q), w\right\rangle+\nu\left\langle\nabla_{q} g_{i}(t, q), v\right\rangle \\
& \geq\left\langle\nabla_{q} g_{i}(t, q)-\nabla_{q} g_{i}\left(t_{0}, q\left(t_{0}\right)\right), w\right\rangle+\nu \delta \\
& \geq \nu \delta-M|w|\left[\left|t-t_{0}\right|+\left|q-q\left(t_{0}\right)\right|\right]
\end{aligned}
$$

and consequently from Assumptions (A3) and (A5)

$$
\partial_{t} g_{i}(t, q)+\left\langle\nabla_{q} g_{i}(t, q), w_{\nu}\right\rangle \geq \nu \delta-(M|w|+M)\left[\left|t-t_{0}\right|+\left|q-q\left(t_{0}\right)\right|\right] .
$$

So for every $\nu>0$, if $(t, q)$ is closed enough to $\left(t_{0}, q\left(t_{0}\right)\right)$, we deduce that for all $i \in I_{\kappa \rho}(t, q) \cap I\left(t_{0}, q\left(t_{0}\right)\right)$

$$
\partial_{t} g_{i}(t, q)+\left\langle\nabla_{q} g_{i}(t, q), w_{\nu}\right\rangle \geq \frac{\nu \delta}{2} .
$$

For the indices $i \notin I_{\kappa \rho}(t, q)$, we have

$$
g_{i}(t, q)+h\left[\partial_{t} g_{i}(t, q)+\left\langle\nabla_{q} g_{i}(t, q), w_{\nu}\right\rangle\right] \geq \kappa \rho-h \beta(1+|w|+\nu) .
$$

Finally for $i \notin I\left(t_{0}, q\left(t_{0}\right)\right)$,

$g_{i}(t, q)+h\left[\partial_{t} g_{i}(t, q)+\left\langle\nabla_{q} g_{i}(t, q), w_{\nu}\right\rangle\right] \geq \sigma-h \beta(1+|w|+\nu)-\beta\left[\left|t-t_{0}\right|+\left|q-q\left(t_{0}\right)\right|\right]$, with

$$
\sigma:=\min _{i \notin I\left(t_{0}, q\left(t_{0}\right)\right)} g_{i}\left(t_{0}, q\left(t_{0}\right)\right)>0 .
$$

We conclude that for each fixed $\nu>0$, there are $\epsilon_{\nu}$ and $h_{\nu}$ such that for every $h<h_{\nu},(t, q) \in U$ with $\left|t-t_{0}\right|+\left|q-q\left(t_{0}\right)\right| \leq \epsilon_{\nu}$ and $q \in Q(t)$ we have

$$
\forall i, \quad g_{i}(t, q)+h\left[\partial_{t} g_{i}(t, q)+\left\langle\nabla_{q} g_{i}(t, q), w_{\nu}\right\rangle\right] \geq h \frac{\nu \delta}{2},
$$

which by a first-order expansion in time gives:

$$
\forall i, \quad g_{i}(t+h, q)+h\left\langle\nabla_{q} g_{i}(t+h, q), w_{\nu}\right\rangle \geq h \frac{\nu \delta}{2}+O_{h \rightarrow 0}(h) .
$$

At the cost of decreasing $h_{\nu}$, it comes for $h<h_{\nu}$,

$$
\forall i, \quad g_{i}(t+h, q)+h\left\langle\nabla_{q} g_{i}(t+h, q), w_{\nu}\right\rangle \geq 0,
$$

and consequently, $w_{\nu} \in K_{h}(t+h, q)$ for every $h<h_{\nu},(t, q) \in U$ with $\left|t-t_{0}\right|+\mid q-$ $q\left(t_{0}\right) \mid \leq \epsilon_{\nu}$ and $q \in Q(t)$.

Step 2. Let us now fix the parameter $\nu$. 
Thanks to the uniform Lipschitz regularity of the maps $q_{h}$ and their uniform convergence towards $q$, there exists $\tilde{h}_{\nu} \leq h_{\nu}$ such that for $\epsilon \leq \epsilon_{\nu} /(2+2 K)$ and $h \leq \tilde{h}_{\nu}$,

$$
t_{h}^{k}, \quad t_{h}^{k+1} \in\left[t_{0}-\epsilon, t_{0}+\epsilon\right] \Rightarrow\left|t_{h}^{k+1}-t_{0}\right|+\left|q_{h}^{k}-q\left(t_{0}\right)\right| \leq \epsilon_{\nu}
$$

From this, as $q_{h}^{k} \in Q\left(t_{h}^{k}\right)$ from Proposition 2.1, the previous step (with $t=t_{h}^{k}$ ) gives $w_{\nu} \in K_{h}\left(t_{h}^{k+1}, q_{h}^{k}\right)$. Therefore, $K_{h}\left(t_{h}^{k+1}, q_{h}^{k}\right)$ being convex, we have

$$
\left\langle u_{h}^{k}+h f_{h}^{k}-u_{h}^{k+1}, w_{\nu}-u_{h}^{k+1}\right\rangle \leq 0
$$

We sum up these inequalities for $k$ from $n$ to $p$, integers chosen such that $t_{h}^{n}$ is the first time step in $\left[t_{0}-\epsilon, t_{0}-\epsilon+h\right]$ and $t_{h}^{p+1}$ the last one in $\left[t_{0}+\epsilon-h, t_{0}+\epsilon\right]$. First, we know that

$$
\left|\sum_{k=n}^{p} h\left\langle f^{k}, w_{\nu}-u_{h}^{k+1}\right\rangle\right| \leq\left(\left|w_{\nu}\right|+K\right) \int_{t_{0}-\epsilon}^{t_{0}+\epsilon} F(t) d t
$$

with $K:=\sup _{h}\left\|u_{h}\right\|_{\infty}$. We also have

$$
\sum_{k=n}^{p}\left\langle u_{h}^{k}-u_{h}^{k+1}, w_{\nu}\right\rangle=\left\langle u_{h}\left(t_{h}^{n}\right)-u_{h}\left(t_{h}^{p+1}\right), w_{\nu}\right\rangle .
$$

We deal with the remainder as follows: we write

$$
\sum_{k=n}^{p}\left\langle u_{h}^{k}-u_{h}^{k+1},-u_{h}^{k+1}\right\rangle=\sum_{k=n}^{p}\left\langle u_{h}^{k}-u_{h}^{k+1}, u_{h}^{k}\right\rangle-\left|u_{h}^{n}\right|^{2}+\left|u_{h}^{p+1}\right|^{2},
$$

which gives

$$
\begin{aligned}
\sum_{k=n}^{p}\left\langle u_{h}^{k}-u_{h}^{k+1},-u_{h}^{k+1}\right\rangle & =\frac{1}{2} \sum_{k=n}^{p}\left|u_{h}^{k}-u_{h}^{k+1}\right|^{2}+\frac{1}{2}\left[-\left|u_{h}\left(t_{h}^{n}\right)\right|^{2}+\left|u_{h}\left(t_{h}^{p+1}\right)\right|^{2}\right] \\
& =\frac{1}{2} \operatorname{Var}_{2}\left(u_{h}\right)_{\left[t_{h}^{n}, t_{h}^{p}\right]}^{2}+\frac{1}{2}\left[-\left|u_{h}\left(t_{h}^{n}\right)\right|^{2}+\left|u_{h}\left(t_{h}^{p}\right)\right|^{2}\right], \quad
\end{aligned}
$$

where we wrote $\operatorname{Var}_{2}$ for the $L^{2}$-variation of a function. Using (3.19)-(3.22), we finally get:

$$
\begin{gathered}
\frac{1}{2} \operatorname{Var}_{2}\left(u_{h}\right)_{\left[t_{h}^{n}, t_{h}^{p}\right]}^{2}+\frac{1}{2}\left[-\left|u_{h}\left(t_{h}^{n}\right)\right|^{2}+\left|u_{h}\left(t_{h}^{p+1}\right)\right|^{2}\right] \\
+\left\langle u_{h}\left(t_{h}^{n}\right)-u_{h}\left(t_{h}^{p}\right), w_{\nu}\right\rangle \lesssim \int_{t_{0}-\epsilon}^{t_{0}+\epsilon} F(t) d t
\end{gathered}
$$

Let us now choose a sequence of $\epsilon_{m}$ going to zero, such that $u_{h}$ pointwisely converges to $u$ at the instants $t_{0}-\epsilon_{m}$ and $t_{0}+\epsilon_{m}$ (which is possible as $u_{h}$ converges almost everywhere towards $u$ ). For each $\epsilon_{m}$ and $h$ small enough, we have shown that the 
last inequality holds. Then, passing to the limit for $h \rightarrow 0$ we get

$$
\begin{gathered}
\frac{1}{2} \operatorname{Var}_{2}(u)_{\left[t_{0}-\epsilon_{m}, t_{0}+\epsilon_{m}\right]}^{2}+\frac{1}{2}\left[-\left|u\left(t_{0}-\epsilon_{m}\right)\right|^{2}+\left|u\left(t_{0}+\epsilon_{m}\right)\right|^{2}\right] \\
+\left\langle u\left(t_{0}-\epsilon_{m}\right)-u\left(t_{0}+\epsilon_{m}\right), w_{\nu}\right\rangle \lesssim \int_{t_{0}-\epsilon_{m}}^{t_{0}+\epsilon_{m}} F(t) d t
\end{gathered}
$$

which gives for $\epsilon_{m} \rightarrow 0$

$$
\frac{1}{2} \operatorname{Var}_{2}(u)_{\left[t_{0}^{-}, t_{0}^{+}\right]}^{2}+\frac{1}{2}\left[-\left|u^{-}\left(t_{0}\right)\right|^{2}+\left|u^{+}\left(t_{0}\right)\right|^{2}\right]+\left\langle u^{-}\left(t_{0}\right)-u^{+}\left(t_{0}\right), w_{\nu}\right\rangle \leq 0 .
$$

Finally we obtain

$$
\frac{1}{2}\left|u^{+}\left(t_{0}\right)-u^{-}\left(t_{0}\right)\right|^{2}+\frac{1}{2}\left[-\left|u^{-}\left(t_{0}\right)\right|^{2}+\left|u^{+}\left(t_{0}\right)\right|^{2}\right]+\left\langle u^{-}\left(t_{0}\right)-u^{+}\left(t_{0}\right), w_{\nu}\right\rangle \leq 0 .
$$

By expanding the square quantities, this can be written as follows

$$
\left\langle u^{-}\left(t_{0}\right)-u^{+}\left(t_{0}\right), w_{\nu}-u^{+}\left(t_{0}\right)\right\rangle \leq 0 .
$$

To conclude the proof, it now suffices to remember that $w_{\nu}=w+\nu v$ and, since for each $\nu>0$, the previous reasoning holds, we obtain (3.17) by letting $\nu$ go to 0 in $(3.23)$.

\section{Application to the Modeling of Inelastic Collisions}

\section{The continuous model}

We consider a mechanical system of $N$ spherical rigid particles in three dimensions. We denote by $q_{i} \in \mathbb{R}^{3}$ the position of the center of particle $i$, by $r_{i}$ its radius, by $m_{i}$ its mass and by $f_{i} \in \mathbb{R}^{3}$ the external force exerted on it. Let $q \in \mathbb{R}^{3 N}$ be defined by $q:=\left(\ldots, q_{i}, \ldots\right)$ and $f \in \mathbb{R}^{3 N}$ by $f:=\left(\ldots, f_{i}, \ldots\right)$. We denote by $D_{i j}(q)$ the signed distance between particles $i$ and $j$ :

$$
D_{i j}(q):=\left|q_{i}-q_{j}\right|-\left(r_{i}+r_{j}\right),
$$

and we set $e_{i j}(q)=\left(q_{j}-q_{i}\right) /\left|q_{j}-q_{i}\right|$ (see Fig. 1).

The problem we are interested in is to describe the path of the configuration $q$ submitted to the force-field $f$ and undergoing inelastic collisions. This inelastic collision law can be modeled by imposing nonoverlapping contraints on the particles

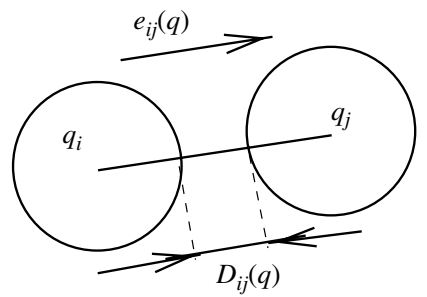

Fig. 1. Particles $i$ and $j$ : notations. 
(see the work of Moreau [17] introducing this concept). Therefore, we write that the positions of the particles have to belong to a set of admissible configurations $Q_{0}$ avoiding overlappings:

$$
q \in Q_{0}:=\bigcap_{i, j}\left\{q, D_{i j}(q) \geq 0\right\} .
$$

We define $M$ as the mass matrix of dimension $3 N \times 3 N, M=\operatorname{diag}(\ldots$, $\left.m_{i}, m_{i}, m_{i}, \ldots\right)$. Then, we denote by $G_{i j} \in \mathbb{R}^{3 N}$ the gradient of distance $D_{i j}$ with respect to the positions of the particles:

$$
G_{i j}(q)=\left(\ldots, 0,-e_{i j}, 0, \ldots, 0, e_{i j}, 0, \ldots, 0\right)^{t} .
$$

The set $\mathcal{C}_{q}$ is the set of admissible velocities:

$$
\mathcal{C}_{q}:=\left\{u,\left\langle G_{i j}(q), u\right\rangle \geq 0, \text { if } D_{i j}(q)=0\right\} .
$$

To finish with notations, we denote by $\lambda=\left(\ldots, \lambda_{i j}, \ldots\right) \in \mathbb{R}^{N(N-1) / 2}$ the vector made of the Lagrange multipliers associated to the $N(N-1) / 2$ constraints " $D_{i j}(q) \geq 0$ ".

Let $I=] 0, T$ [ be the time interval. The multi-particle model we are interested in may be formally phrased as follows:

$$
\left\{\begin{array}{l}
q \in W^{1, \infty}\left(I, \mathbb{R}^{3 N}\right), \quad \dot{q} \in B V\left(I, \mathbb{R}^{3 N}\right), \quad \lambda \in\left(\mathcal{M}_{+}(I)\right)^{N(N-1) / 2}, \\
\forall t \in I, \quad \dot{q}\left(t^{+}\right)=P_{\mathcal{C}_{q(t)}} \dot{q}\left(t^{-}\right), \\
M \ddot{q}=f+\sum_{i<j} \lambda_{i j} G_{i j}(q) \\
\operatorname{supp}\left(\lambda_{i j}\right) \subset\left\{t, D_{i j}(q(t))=0\right\} \quad \text { for all } i, j \\
D_{i j}(q(t)) \geq 0 \quad \text { for all } i, j \\
q(0)=q^{0} \text { such that } D_{i j}\left(q^{0}\right)>0 \quad \text { for all } i, j, \dot{q}(0)=u^{0} .
\end{array}\right.
$$

The main equation

$$
M \ddot{q}-f=\sum_{i<j} \lambda_{i j} G_{i j}(q) \in-N\left(Q_{0}, q\right)
$$

expresses the fact that overlapping is prevented by a repulsive force (the impulsion) acting on each sphere along the normal vector at the contact point. When there is no contact, $N\left(Q_{0}, q\right)$ is reduced to $\{0\}$, so that (4.3) reads as $M \ddot{q}=f$, which is the Fundamental Principle of Dynamics applied on each sphere. Equation $\dot{q}\left(t^{+}\right)=$ $P_{\mathcal{C}_{q(t)}} \dot{q}\left(t^{-}\right)$provides the inelastic collision model. It can be extended to an elastic collision model with a restitution coefficient $e$ by writing

$$
\dot{q}\left(t^{+}\right)=P_{\mathcal{C}_{q(t)}} \dot{q}\left(t^{-}\right)-e P_{N\left(Q_{0}, q(t)\right)} \dot{q}\left(t^{-}\right) .
$$

We assume for simplicity that each mass $m_{i}$ is equal to 1 . Then Problem (4.2) fits into the previously studied framework. 
Remark 4.1. The case of different masses can be taken into account by using the adapted scalar product $(u, v)_{M}=\langle M u, v\rangle$, as was done in [10]. It turns back to replace the projection step in the numerical algorithm by

$$
u^{n+1}=P_{K_{h}\left(t_{h}^{n+1}, q_{h}^{n}\right)}\left(u^{n}+h M^{-1} f^{n}\right),
$$

where $P$ here denotes the projection relatively to this new norm.

$M$ being a diagonal matrix with non-negative diagonal coefficients, it is easy to show that the following results still hold true in that case.

We emphasize that Assumption (A0) is satisfied as soon as

$$
\min _{i} r_{i}>0 \text {, }
$$

and then Assumptions (A1) and (A4) hold true.

In order to apply our previous results, it remains to check Assumption (A6). As explained in [30], that corresponds to estimate the Kuhn-Tucker multipliers. Such an estimate is given in the following lemma.

Lemma 4.2. There exists $a>0$ (depending on $N$ and on the radii $r_{i}$ ) such that for all $q \in \mathbb{R}^{3 N}, F \in \mathbb{R}^{3 N}$ and Lagrange multipliers $\left(\mu_{i j}\right) \in \mathbb{R}^{N(N-1) / 2}$ satisfying

$$
\sum \mu_{i j} G_{i j}(q)=F \text { with } \mu_{i j} \geq 0 \text { and } \mu_{i j}=0 \text { when } D_{i j}(q)>0,
$$

then

$$
\mu_{i j} \leq a|F|
$$

Concerning the proof of this lemma, we refer the reader to Proposition 4.7 of [30] (for a geometric proof) and to Proposition 2.18 of [11] (for a more "physical" proof). These proofs are written in a two-dimensional framework but they can be easily extended in our three-dimensional case. Actually, Lemma 4.2 is equivalent to Assumption (A6) with $\rho=0$. However, it can be extended and still holds for $\rho$ small enough (for example $\rho<\inf _{i} r_{i}$ ), see Remark 4.11 of [30]. Consequently, Assumption (A6) is satisfied for some small enough $\rho>0$.

According to our main theorem (Theorem 1.3), it follows that Problem (4.2) has solutions and that the associated numerical scheme converges (up to a subsequence).

We can allow the radii to depend on time. In that case, the set of admissible velocities can be written

$$
\mathcal{C}_{t, q}:=\left\{u,\left\langle G_{i j}(q), u\right\rangle \geq 0, \text { if } D_{i j}(t, q)=0\right\},
$$

where

$$
D_{i j}(t, q)=\left|q_{i}-q_{j}\right|-\left(r_{i}(t)+r_{j}(t)\right) .
$$

As soon as $r_{i}$ is uniformly twice-differentiable in time and

$$
\inf _{t \in[0, T]} \inf _{i} r_{i}(t)>0,
$$

Theorem 1.3 gives the existence of solutions to the continuous problem and the convergence of the scheme up to a subsequence. 
These theoretical results permit to legitimate the implementation of this numerical scheme. This was performed by the second author by creating SCoPI Software [29]. We refer the reader to [10] for some good properties of stability and robustness for the algorithm and efficiency for large time steps.

Remark 4.3. We refer the reader to [9], where the second author extends this model in order to consider gluey particles. In this case, she add an extra parameter (depending on $q$ ) for describing the corresponding admissible set. This new operation does not keep the necessary regularity of the admissible set. She has already obtained a result of convergence for the associated numerical scheme in the singleconstraint case. We plan in a forthcoming work to extend this proof with the ideas presented here in order to deal with the multi-constraint case.

\section{References}

1. P. Ballard, The dynamics of discrete mechanical systems with perfect unilateral constraints, Arch. Rational Mech. Anal. 154 (2000) 199-274.

2. F. Bernicot and J. Venel, Stochastic perturbations of sweeping process, submitted, arXiv:1001.3128.

3. F. Bernicot and J. Venel, Existence of solutions for second-order differential inclusions involving proximal normal cones, submitted, arXiv:1006.2292.

4. F. H. Clarke, R. J. Stern and P. R. Wolenski, Proximal smoothness and the lower- $C^{2}$ property, J. Convex Anal. 2 (1995) 117-144.

5. F. H. Clarke, Y. S. Ledyaev, R. J. Stern and P. R. Wolenski, Nonsmooth Analysis and Control Theory (Springer-Verlag, 1998).

6. G. Colombo and M. D. P. Monteiro Marques, Sweeping by a continuous prox-regular set, J. Differential Equation 187 (2003) 46-62.

7. R. Dzonou and M. D. P. Monteiro Marques, A sweeping process approach to inelastic contact problems with general inertia operators, Eur. J. Mech. A/Solids 26 (2007) 474-490.

8. R. Dzonou, M. D. P. Monteiro Marques and L. Paoli, A convergence result for a vibroimpact problem with a general inertia operator, Nonlinear Dyn. 58 (2009) 361-384.

9. A. Lefebvre, Numerical simulation of gluey particles, Model. Math. Anal. Numer. 43 (2009) 53-80.

10. B. Maury, A time-stepping scheme for inelastic collisions, Numer. Math. 102 (2006) 649-679.

11. B. Maury and J. Venel, A discrete contact model for crowd motion, Model. Math. Anal. Numer., arXiv:0901.0984.

12. M. D. P. Monteiro-Marques, Differential Inclusions in Non-smooth Mechanical Problems: Shocks and Dry Friction, PNLDE, Vol. 9 (Birkhäuser, 1993).

13. M. D. P. Monteiro-Marques and L. Paoli, An existence result in non-smooth dynamics, in Nonsmooth Mechanics and Analysis, Adv. Mech. Math., Vol. 12 (Springer, 2006), Chap. 23, pp. 279-288.

14. J. J. Moreau, Décomposition orthogonale d'un espace Hilbertien selon deux cônes mutuellement polaires, C. R. Acad. Sci. Ser. I 255 (1962) 238-240.

15. J. J. Moreau, Liaisons unilatérales sans frottements et chocs inélastiques, C. R. Acad. Sci. Ser. II 296 (1983) 1473-1476.

16. J. J. Moreau, Standard Inelastic Shocks and the Dynamics of Unilateral Constraints, CISM Courses and Lectures, Vol. 288 (Springer, 1985), pp. 173-221. 
17. J. J. Moreau, Some numerical methods in multibody dynamics: Application to granular materials, Eur. J. Mech. A/Solids 13 (1994) 93-114.

18. L. Paoli and M. Schatzman, Mouvement à un nombre fini de degrés de liberté avec contraintes unilatérales; cas avec perte d'énergie, Model. Math. Anal. Numer. 27-6 (1993) 673-717.

19. L. Paoli and M. Schatzman, Penalty approximation for non smooth constraints in vibro-impact, J. Differential Equations 177 (2001) 375-418.

20. L. Paoli and M. Schatzman, A numerical scheme for impact problems I and II, SIAM J. Numer. Anal. 40-2 (2002) 702-768.

21. L. Paoli and M. Schatzman, Penalty approximation for dynamical systems submitted to multiple non smooth constraints, Multibody Syst. Dynam. 8 (2002) 347-366.

22. L. Paoli, An existence result for non-smooth vibro-impact problems, J. Differential Equations 211 (2005) 247-281.

23. L. Paoli, Time-stepping approximation of rigid-body dynamics with perfect unilateral constraints. I. The inelastic impact case, A.R.M.A., to appear.

24. L. Paoli, Time-stepping approximation of rigid-body dynamics with perfect unilateral constraints. II. The partially inelastic impact case, A.R.M.A., to appear.

25. R. A. Poliquin, R. T. Rockafellar and L. Thibault, Local differentiability of distance functions, Trans. Amer. Math. Soc. 352 (2000) 5231-5249.

26. M. Schatzman, A class of nonlinear differential equations of second order in time, Nonlinear Anal. 2 (1978) 355-373.

27. M. Schatzman, Uniqueness and continuous dependence on data for one-dimensional impact problems, Math. Comput. Model. 28 (1998) 1-18.

28. M. Schatzman, Penalty method for impact in generalized coordinates, Phil. Trans. Roy. Soc. London A 359 (2001) 2429-2446.

29. SCoPI Software, presentation available at http://www.projet-plume.org/relier/scopi, and numerical simulations available at http://www.cmap.polytechnique.fr/ lefebvre/ SCoPI.htm

30. J. Venel, A numerical scheme for a class of sweeping processes, Num. Math. (2011), arXiv:0904.2694. 